\title{
Variación estacional de la estructura comunitaria de peces en dos arrecifes: rocoso-coralino y de octocorales, en el Bajo Las Caracas, Venezuela
}

\author{
Seasonal variations of the community structure of fishes in two reefs: rocks-stone \\ corals and octocorals, in Bajo Las Caracas, Venezuela \\ Ángel Rafael Fariña Pestano ${ }^{1}$ y Elizabeth Méndez De Elgueazabal ${ }^{1}$ \\ ${ }^{1}$ Departamento de Biología, Escuela de Ciencias, Universidad de Oriente, Núcleo de Sucre. \\ Cumaná 6101, Estado Sucre, Venezuela \\ afarina46@yahoo.com
}

\begin{abstract}
We studied the seasonal variation of ichtyofauna on two reefs: a stony coral reef and an octocoral (soft coral) reef. Monthly visual censuses were implemented based on transects of $40 \times 2 \mathrm{~m}\left(80 \mathrm{~m}^{2}\right)$ placed in each ecosystem. The results indicate correlation patterns between annual changes in the fish community and the physical seasonal parameters. Such patterns occur principally within the soft coral reef, where the species richness and relative abundance are greatest during the months of upwelling. The observed groups of the pelagic species Sardinella aurita and Decapterus punctatus are responsible for the decreased diversity during the upwelling peaks. In the stony coral reef, species richness was positively correlated with visibility and abundance, coinciding with records of similar reefs in nearby areas. Comparing the monthly abundance of ichtyofauna, the dispersion in the data from the octocorals was greater compared to the stony coral reef. Coupled with the low number of constant species, this demonstrates that the ichthyofauna associated with the gorgonian make less frequent use of this ecosystem. Though the two reefs are adjacent to one another, nine species regularly present in the stony coral reef were not observed in the soft coral reef. This pattern conforms to the deterministic assumptions regarding the use of space and biodiversity in reef areas. In conclusion, the results reinforce that the octocoral reef is a particularly distinct habitat within the diversity of ecosystems in the Venezuelan submarine environment.
\end{abstract}

Key words: Upwelling, Caribbean, ichthyofauna, Sardinella aurita, Aulostomus maculatus

\section{Introducción}

La estructura de las comunidades ícticas ha sido asociada a las características de su hábitat, como lo señalan los estudios de Grigg (1994) en Hawai, Caley \& St John (1996) en regiones tropicales, Garpe \& Oehman (2003) en Tanzania y Bouchon-Navarro et al. (2005) en el Caribe. Estos últimos autores, evaluaron la ictiofauna desde República Dominicana hasta Bequia (al norte de Grenada),
Resumen.- Se estudió la variación estacional de la ictiofauna en dos arrecifes: uno rocoso coralino (pétreo) y otro de octocorales. Se aplicaron censos visuales, mensualmente, en transectos de $40 \times 2 \mathrm{~m}\left(80 \mathrm{~m}^{2}\right)$ para cada ecosistema. Los resultados indican patrones de correlación entre la variación anual de la comunidad de peces y los parámetros físicos estacionales, principalmente en el arrecife de corales blandos, donde la mayor riqueza de especies y abundancias relativas suceden en los meses de surgencia. La ocurrencia de cardúmenes pelágicos de Sardinella aurita y Decapterus punctatus serían responsables del descenso en la diversidad durante los máximos de surgencia. En el arrecife pétreo la riqueza se correlacionó positivamente con la visibilidad y la abundancia, coincidiendo con lo registrado en arrecifes similares cercanos. Al comparar las abundancias mensuales de los peces, se observó una mayor dispersión de los datos en los octocorales que en el arrecife pétreo, lo cual, unido al bajo número de especies constantes, evidencia que la ictiofauna asociada a los gorgonios hace un uso menos frecuente de este ecosistema. A pesar de estar adyacentes los dos sustratos, nueve especies que se presentaron de manera constante en el arrecife rocoso coralino no fueron observadas en los corales blandos, concordando con las hipótesis determinísticas sobre el uso del espacio y la biodiversidad en arrecifes. Se refuerza el planteamiento que propone al arrecife de octocorales como un hábitat particularmente distinto dentro de la diversidad de ecosistemas submarinos venezolanos.

Palabras clave: Surgencia, Caribe, ictiofauna, Sardinella aurita, Aulostomus maculatus

siguiendo la línea de las Antillas, y encontraron que la composición de las comunidades de peces pareciera estar influenciada principalmente por un gradiente hidrológico y latitudinal, así como por el tipo de hábitat. Beukers \& Jones (1998) indicaron que la complejidad del sustrato puede disminuir el efecto de los piscívoros sobre la ictiofauna local.

Varios estudios han considerado la conformación 
estructural de arrecifes coralinos o rocosos coralinos. No obstante, en zonas tropicales hay áreas importantes donde sólo existen corales blandos (octocorales), los cuales ofrecen un hábitat que puede ser ocupado por comunidades con una gran riqueza de especies, sirviendo además como refugio para peces (Risk et al. 1998). Las diferencias en la estructura física de un arrecife de corales blandos respecto a los arrecifes pétreos, así como las defensas químicas de los octocorales, hacen que la ocupación del espacio por los peces sea igualmente distinta, con importantes variaciones respecto a la composición comunitaria de la ictiofauna entre ambos sistemas (Fariña et al. 2008).

En Venezuela, además de existir una multiplicidad de hábitats que incluyen los tipos de arrecifes citados anteriormente, se presentan dos estaciones: lluviosa y seca, al igual que un fenómeno anual de surgencia costera, particularmente evidente en la región nororiental, el cual está determinado principalmente por el efecto de los vientos alisios del noreste. Estos vientos comienzan a incidir de manera medianamente constante en noviembre y diciembre, permaneciendo aproximadamente hasta abril (Okuda 1978, Ferraz-Reyes 1989). Dicho proceso, unido a los aportes del río Orinoco, genera un impacto importante en la estructura comunitaria de peces (Cervigón 2005), por lo que resulta necesaria la evaluación estacional de los cambios en la riqueza de especies y en la abundancia íctica.

La influencia de las surgencias sobre la estructura comunitaria de la ictiofauna ha sido registrada en áreas coralinas de distintas zonas geográficas. RamírezGutiérrez et al. (2007) encontraron fluctuaciones estacionales en la comunidad de peces, relacionadas con la surgencia en el Golfo de Tehuantepec, México. También en México, Pérez-España et al. (1996) registraron cambios en la estructura comunitaria de peces de arrecifes entre las estaciones del año y entre los sitios de estudio.

En la presente investigación se evaluó la estructura comunitaria íctica y su variación estacional de manera comparativa, en dos sustratos de arrecifes adyacentes, uno dominado por octocorales y el otro rocoso con corales pétreos, ubicados en el Bajo Las Caracas, un promontorio submarino que no había sido estudiado previamente.

\section{Material y métodos}

\section{Descripción del área de estudio}

El Bajo Las Caracas es una meseta submarina que emerge a unos $3 \mathrm{~km}$ al norte de la Isla Caracas Oeste $\left(10^{\circ} 23^{\prime} 42^{\prime \prime} \mathrm{N}\right.$ y los $64^{\circ} 26^{\prime} 42^{\prime \prime W}$ ) y abarca una extensión aproximada de
$4 \mathrm{~km}^{2}$. A pesar de que no existen registros en la zona, visitas recurrentes al lugar han constatado la existencia de corrientes estacionales en dirección oeste-este (Fariña obs. pers.). La visibilidad vertical es de más de $10 \mathrm{~m}$ durante casi todo el año, pero disminuye considerablemente en la época de surgencia costera, entre diciembre y abril.

Para el muestreo y la toma de datos se escogió un sistema ecológico constituido por dos estructuras arrecifales que se ubican de manera adyacente y paralelas a la costa, separadas por unos $5 \mathrm{~m}$ de distancia. La primera de estas estructuras es un arrecife pétreo, conformado por un promontorio de piedras con corales escleractinios, con numerosas oquedades, por lo que se puede considerar estructuralmente compleja. Su profundidad máxima es de $6 \mathrm{~m}$, pero presenta rocas altas $(2-3 \mathrm{~m})$ que llegan hasta unos $3 \mathrm{~m}$ de la superficie. La segunda estructura es un arrecife de octocorales, sin formaciones pétreas, que se desarrolla sobre una plataforma de sustrato duro.

\section{Parámetros ambientales}

La temperatura superficial del agua se registró utilizando un termómetro de mercurio y la salinidad mediante el empleo de un refractómetro manual. Se estimó la transparencia del agua mediante una medida referencial de la visibilidad horizontal. Para ello, se utilizó el apoyo de un buzo que sostuvo una placa blanca al extremo de una cuerda y se procedió a nadar hasta donde dicha placa dejase de verse. Para el oleaje y la intensidad del viento se utilizó la escala Beaufort (CENAHP 2007). Igualmente, se determinó la dirección del viento con una veleta ya que en el área de muestreo incide desde el noroeste y desde el noreste, dependiendo de la época del año; así como la ocurrencia de fenómenos de mar de fondo, los cuales también suceden estacionalmente en la zona. Los dos arrecifes considerados en este estudio, están muy cerca entre sí, por lo que las variables ambientales fueron tomadas en el área y utilizadas en los análisis ícticos de ambos sistemas.

\section{Muestreo y obtención de datos}

Entre julio de 2005 y junio de 2006 se realizaron censos visuales mensuales en dos transectos, uno en cada arrecife. Se utilizó una modificación del protocolo Atlantic and Gulf Rapid Reef Assessment (AGRRA) (Ginsburg 2000), que incluye el conteo de todas las especies observadas. Igualmente, la longitud de los transectos fue ampliada a $40 \times 2 \mathrm{~m}$, respecto a la establecida por AGRRA, para abarcar un área representativa del arrecife. Los censos de peces se realizaron mensualmente entre las 08:00 y 09:00 h, el mismo día para ambos ecosistemas, mediante buceo (libre). Se tomaron fotografías de las 
especies de peces y de corales, así como videos submarinos en el recorrido del transecto. La identificación de los peces se realizó utilizando las descripciones de Humann \& Deloach (2002). En pocos casos particulares, cuando la determinación taxonómica no fue posible a nivel visual, se anotaron las características de la especie, se registró el número de individuos y posteriormente se capturaron ejemplares con técnicas diversas (arpón, anzuelo, malla) para el uso de claves (Cervigón 1991, 1993, 1994, 1996).

\section{Índices ecológicos y análisis estadísticos}

Se calculó la diversidad de Shannon-Weaver y la equitabilidad. La riqueza se expresó como número de especies. Para determinar la frecuencia de aparición de las especies en cada transecto, se empleó la fórmula de constancia (C) de Bohdenheiner \& Balogh (Krebs 1972). Las especies se clasificaron en constantes (C $>50 \%)$, accesorias $(25 \% \leq C \leq 50 \%)$ y accidentales $(C<25 \%)$. $\mathrm{La}$ abundancia mensual relativa para cada arrecife se expresó en términos de porcentaje, relacionando el número de individuos en cada mes con el total de ejemplares censados en el año.

Debido a que los datos no presentaban normalidad en su distribución, se procedió a realizar un análisis no paramétrico de Kruskall-Wallis, mediante el programa Statgraphics Plus 4.1, para comparar los promedios anuales de la diversidad, la equitabilidad, la riqueza y la abundancia de las especies de peces entre los dos arrecifes.

Para determinar posibles relaciones entre los parámetros ambientales y los descriptores comunitarios de la fauna íctica (abundancia relativa, la diversidad, la equitabilidad y la riqueza), se realizaron correlaciones de Spearman (SPSS 12.0) y análisis de componentes principales (ACP) colocando los meses como casos (Servicio de Computación Kovac 2006), para cada arrecife. Debido a la heterogeneidad de las variables, los datos fueron convertidos a raíz cuadrada y estandarizados utilizando una matriz de correlación, de acuerdo con las recomendaciones del programa MVSP. Se asumió como 0 cuando el viento provino del noreste (alisios del noreste) y 1 cuando incidió desde el noroeste. La presencia de mar de fondo al momento del muestreo se registró con el número 1 y su ausencia como 0 .

Para visualizar la variación de las abundancias de las especies a través del año y entre los arrecifes, se aplicó un análisis de componentes principales (ACP) para cada ecosistema, mediante el programa MVSP, considerando los meses como los casos y a las especies como variables, descartando las pelágicas así como aquellas cuyas abundancias representaron menos del $10 \%$ de la abundancia total.

\section{Resultados}

La temperatura osciló entre $24^{\circ} \mathrm{C}$ y $29^{\circ} \mathrm{C}$, con un promedio anual de $27,25^{\circ} \mathrm{C}$. Los menores valores se registraron en enero, diciembre y febrero. La salinidad no mostró fluctuaciones importantes durante el año y se presentó en un rango entre 34-36, con un valor medio de 35,25. La visibilidad horizontal fluctuó entre $6 \mathrm{~m}$ y $17 \mathrm{~m}$, cuyo promedio fue de 13,5 m; sin embargo, hubo aguas claras durante todo el muestreo. En cinco muestreos realizados, de 5 meses distintos (julio, agosto, noviembre y diciembre de 2006 y febrero de 2007), se registraron vientos del noroeste, lo cual no es común en la zona. Sólo el mes de noviembre, coincidió con un evento de mar de fondo ('rebozo'). Los otros meses fueron, de acuerdo con la escala Beaufort, de calma total, de brisa muy débil (grado 2) o de una brisa moderada (grado 4).

Para los dos arrecifes evaluados, se registró un total de 5.655 peces pertenecientes a 32 familias, 48 géneros y 84 especies.

\section{Arrecife pétreo}

En el arrecife pétreo se censó un total de 4.907 individuos de 31 familias, 48 géneros y 80 especies. El menor número de especies se presentó en diciembre (32) y el mayor en agosto (42) (Fig. 1), con un valor promedio de 37 especies. La mayor abundancia relativa se presentó en marzo (12,45\%) y la más baja en diciembre (5,03\%) (Fig. 2). La diversidad (H') en el arrecife pétreo fluctuó entre 3,47 bits ind. ${ }^{-1}$ en mayo y 4,40 bits ind.$^{-1}$ en agosto, con un promedio anual de 4,00 bits ind. ${ }^{-1}$ (Fig. 3).

El análisis de componentes principales mostró que los 3 primeros ejes contribuían en más de un $75 \%$ con la variabilidad de los datos (Tabla 1). Se encontraron correlaciones significativas positivas de la riqueza con la visibilidad (rs=0,709) y la abundancia ( $(\mathrm{rs}=0,684)$ y entre la diversidad de Shannon-Weaver y la equitatividad (rs= 0,874 ). Hubo una correlación significativa negativa entre la abundancia relativa y la diversidad de Shannon-Weaver $(r s=-0,666)$. La dirección del viento se correlacionó positivamente con el valor Beaufort (rs= 0,877) (Fig. 4).

El resultado del análisis de componentes principales que evalúa el nivel de asociación de los meses, respecto a las abundancias relativas de las especies, agrupó la mayoría de los meses excepto febrero, junio y julio. Entre éstos, los dos primeros meses fueron los más disímiles del resto del año (Fig. 5). Las especies que más aportaron a dicha variación fueron Haemulon chrysargyreum, Thalassoma bifasciatum y Lutjanus griseus (Tabla 2). 


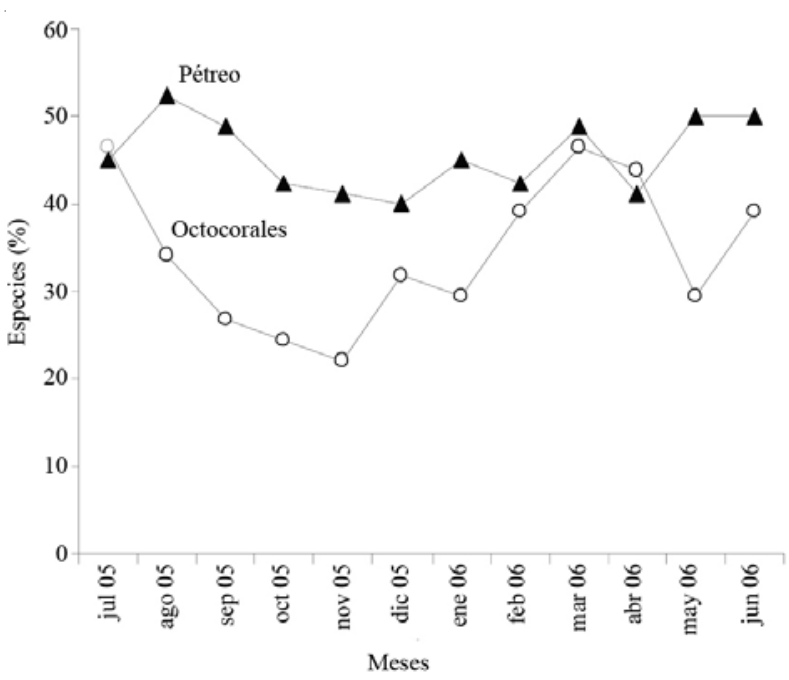

Figura 1

Variación mensual del número de especies en dos arrecifes: pétreo y de octocorales, entre julio de 2005 y junio de 2006, en el Bajo Las Caracas

Monthly variation of the species count in two reefs, stony and octocorals, between July 2005 and June 2006, in Bajo Las Caracas

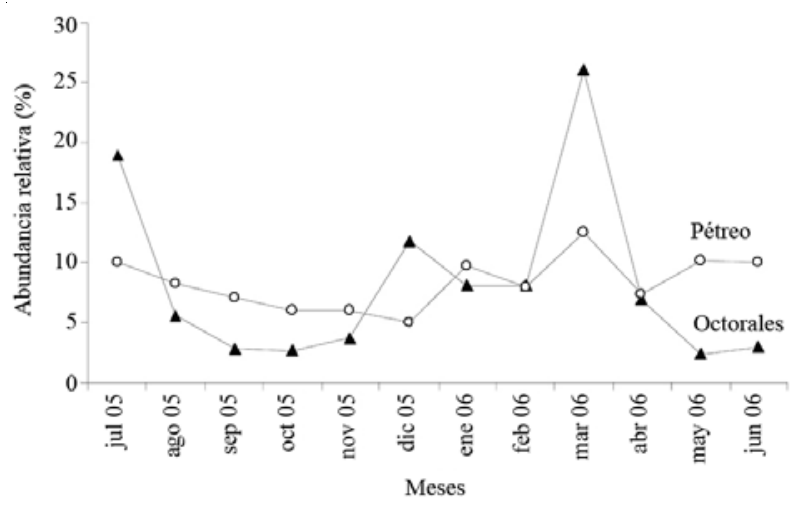

Figura 2

Variación mensual de la abundancia relativa de peces en dos arrecifes: pétreo y de octocorales, entre julio de 2005 y junio de 2006, en el Bajo Las Caracas

Monthly variation in the relative abundance of fish in two reefs, stony and octocorals, between July 2005 and June 2006, in Bajo Las Caracas

Al estudiar la presencia de las especies durante todo el período de muestreo en el arrecife rocoso coralino, se encontró que un 41,35\% de ellas hicieron uso accidental del espacio, 38,7\% fueron residentes constantes y $20 \%$ accesorias. Un número de 18 especies se presentaron durante todos los meses de muestreo (100\% de

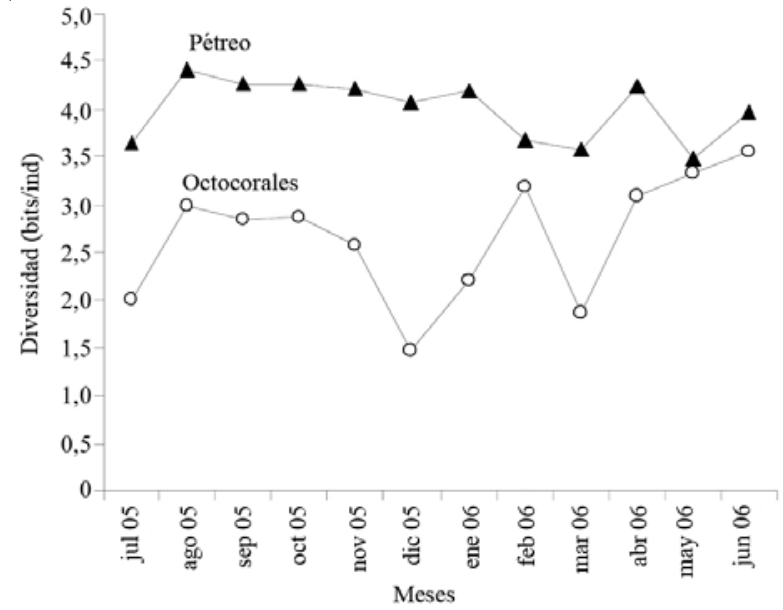

Figura 3

Variación mensual de la diversidad en dos arrecifes: pétreo y de octocorales, entre julio de 2005 y junio de 2006, en el Bajo Las Caracas

Monthly variation of the diversity in two reefs, stony coral and octocorals reefs, between July 2005 and June 2006 in

Bajo Las Caracas

constancia): Acanthemblemaria sp., Acanthurus bahianus, Acanthurus coeruleus, Cantherhines pullus, Chaetodon capistratus, Chaetodon sedentarius, Haemulon chrysargyreum, Haemulon flavolineatum, Holocentrus adscencionis, Microspathodon chrysurus, Odontoscion dentex, Scarus iseri, Scarus taeniopterus, Scarus vetula, Sparisoma aurofrenatum, Sparisoma viride, Stegastes partitus y Thalassoma bifasciatum.

\section{Arrecife de octocorales}

Se contabilizaron 1.073 individuos de 19 familias, 25 géneros y 41 especies. El menor número de especies se registró en noviembre (9) y el mayor en marzo y julio (19), con un promedio de 14 (Fig. 1). Por otro lado, la mayor abundancia mensual relativa en los octocorales también sucedió en marzo (26,0\%) mientras que la menor se observó en mayo (2,42\%) (Fig. 2). La menor diversidad se obtuvo en diciembre (1,47 bits ind. $\left.{ }^{-1}\right)$ y la mayor en junio (3,55 bits ind. $\left.{ }^{-1}\right)$, para un promedio en el año de 2,67 bits ind. ${ }^{-1}$ (Fig. 3).

El análisis de componentes principales mostró que los tres primeros ejes contribuían en más de un 79\% con la variabilidad de los datos (Tabla 1). Se encontraron correlaciones significativas y positivas de la visibilidad con la diversidad de Shannon-Weaver (rs=0,814) y con la equitatividad ( $r s=0,763$ ), entre la riqueza y la abundancia 


\section{Tabla 1}

Autovalores de los análisis de componentes principales para relacionar variables ambientales y parámetros comunitarios a través de los meses del año

Eigen-values from the principal components analysis used to relate environmental variables and community parameters with the months of year

\begin{tabular}{lrrrrrr}
\hline & Eje 1 & Eje 2 & Eje 3 & Eje 1 & Eje 2 & Eje 3 \\
\hline & \multicolumn{3}{c}{ Arrecife pétreo } & \multicolumn{3}{c}{ Arrecife de octocorales } \\
Autovalores & 3,37 & 2,57 & 1,63 & 3,95 & 1,76 & 1,42 \\
Porcentaje & 33,66 & 25,69 & 16,31 & 43,90 & 19,59 & 15,82 \\
Porcentaje acumulado & 33,66 & 59,35 & 75,66 & 43,90 & 63,49 & 79,31 \\
\hline
\end{tabular}
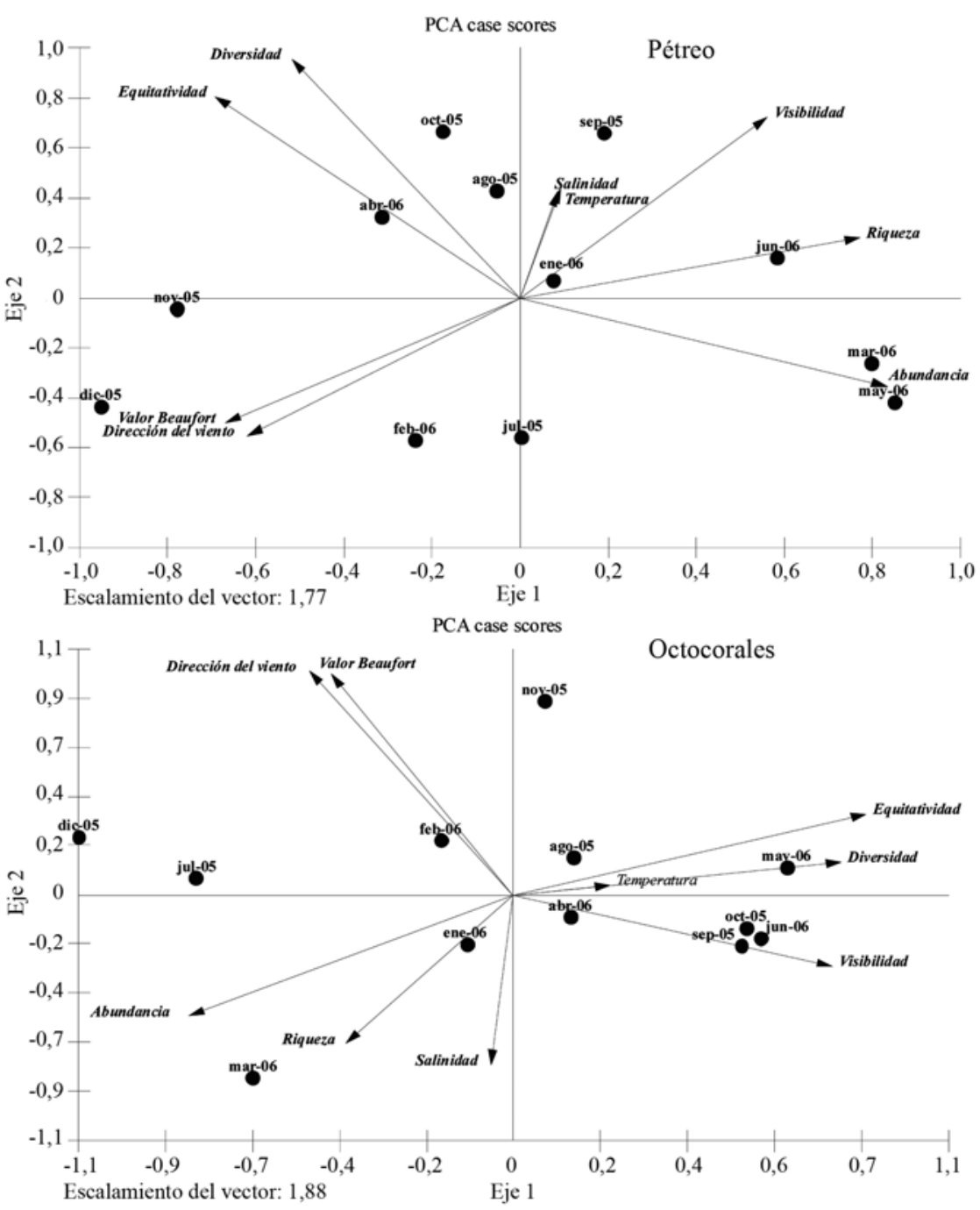

Figura 4

Análisis de componentes principales para relacionar los parámetros ambientales y comunitarios a través de los meses del año, en dos arrecifes del Bajo Las Caracas

Principal components analysis used to relate the environmental and community parameters with the months of the year in two reefs found in Bajo Las Caracas 

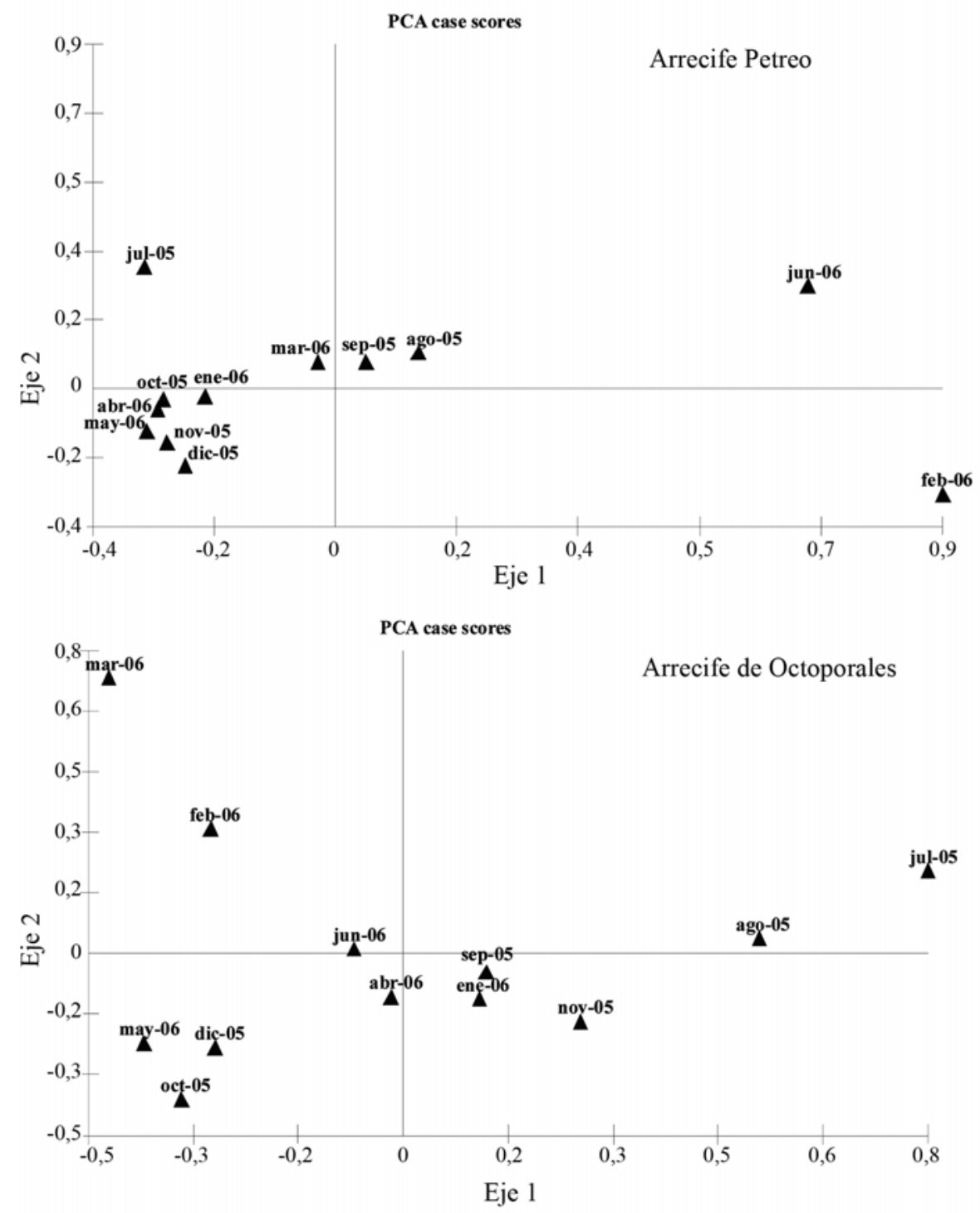

Figura 5

Análisis de componentes principales para relacionar los meses de muestreo con las abundancias de peces no pelágicos en dos arrecifes del Bajo Las Caracas

Relative monthly importance of the non-pelagic species and scatter plot of the principal components analysis used to relate the months of sampling to the abundances of non-pelagic fish in two reefs found in Bajo Las Caracas

(rs=0,696), entre la diversidad y la equitatividad (rs=0,924) así como entre la dirección del viento y el valor Beaufort (rs=0,866). Adicionalmente, se verificó correlación significativa negativa de la abundancia relativa con la diversidad Shannon-Weaver ( $r s=-0,715)$ y con la equitatividad (rs=-0,862). Los meses de diciembre, enero, febrero, marzo y julio, tienden a separarse del resto de los meses del año, coincidiendo con la mayor abundancia y riqueza de peces, así como los mayores valores Beaufort (Fig. 4).
El análisis de componentes principales que asocia los meses del año respecto a la abundancia relativa de las especies indica como grupos aparte a los meses de febrero y marzo, y a los de julio y agosto; otro grupo abarca a mayo, octubre y diciembre, y otro disperso que relaciona al resto de los meses (Fig. 5). En este caso, las especies con mayor influencia en la variación fueron Sparisoma aurofrenatum, Haemulon aurolineatum y Halichoeres bivittatus (Tabla 2). 
Tabla 2

Autovalores de los análisis de componentes principales para estudiar la asociación de los meses respecto a las abundancias de las especies no pelágicas, en dos arrecifes del Bajo Las Caracas

Eigen-values from principal components analysis recorded to study the association of the months of the year with the abundance of non-pelagic species in two reefs found in Bajo Las Caracas

\begin{tabular}{|c|c|c|c|c|c|c|c|}
\hline \multicolumn{5}{|c|}{ Arrecife pétreo } & \multicolumn{3}{|c|}{ Arrecife de octocorales } \\
\hline & Eje 1 & Eje 2 & Eje 3 & & Eje 1 & Eje 2 & Eje 3 \\
\hline Autovalores & 1,81 & 0,35 & 0,21 & & 1,69 & 1,02 & 0,55 \\
\hline Porcentaje & 67,50 & 13,17 & 7,73 & & 38,19 & 22,97 & 12,41 \\
\hline Porcentaje acumulado & 67,50 & 80,67 & 88,40 & & 38,19 & 61,15 & 73,56 \\
\hline Variables & & & & Variables & & & \\
\hline Abudefduf saxatilis & 0,016 & 0,08 & $-0,01$ & Acanthurus bahianus & 0,1 & 0,075 & 0,23 \\
\hline Acanthemblemaria rivasi & $-0,009$ & 0,006 & $-0,026$ & Aulostomus maculatus & 0,017 & 0,077 & $-0,075$ \\
\hline Acanthurus bahiamus & $-0,03$ & 0,076 & $-0,11$ & Cantherhines pullas & 0,055 & 0,033 & $-0,094$ \\
\hline Acanthurus coeruleus & 0,137 & $-0,053$ & 0,084 & Canthigaster rostrata & 0,077 & 0,018 & $-0,02$ \\
\hline Aulostomus maculatus & $-0,027$ & 0,044 & $-0,146$ & Chaetodon capistratus & 0,128 & 0,098 & $-0,279$ \\
\hline Cantherhines pullus & 0,004 & $-0,002$ & $-0,026$ & Haemulon aurolineatum & $-0,173$ & 0,611 & $-0,32$ \\
\hline Chaetodon capistratus & 0,024 & 0,093 & $-0,215$ & Haemulon chrysargyreum & $-0,171$ & $\begin{array}{l}-0,47 \\
-\end{array}$ & $-0,032$ \\
\hline Chaetodon sedentarius & 0,011 & 0,039 & 0,008 & Haemulon steindachneri & 0,239 & 0,026 & $-0,19$ \\
\hline Haemulon carbonarium & 0,011 & 0,083 & 0,076 & Halichoeres bivittatus & 0,023 & 0,098 & 0,509 \\
\hline Haemulon chrysargyreum & 0,981 & 0,055 & $-0,011$ & Scarus iseri & $-0,076$ & 0,232 & $-0,022$ \\
\hline Haemulon flavolineatum & 0,017 & $-0,058$ & $-0,036$ & Scarus taeniopterus & 0,075 & 0,102 & $-0,03$ \\
\hline Halichoeres bivittatus & 0,004 & 0,042 & 0,008 & Serranus tigrinus & 0,035 & 0,095 & 0,1 \\
\hline Holocentrus adscencionis & 0,007 & $-0,026$ & $-0,024$ & Sparisoma aurofrenatum & 0,86 & 0,194 & 0,07 \\
\hline Lutjamus griseus & $-0,026$ & $-0,188$ & 0,806 & Sparisoma chrysopterum & $-0,193$ & 0,264 & $-0,275$ \\
\hline Mulloidichthys martinicus & 0,074 & $-0,033$ & 0,016 & Sparisoma viride & $-0,227$ & 0,392 & 0,587 \\
\hline Microspathodon chrysurus & 0,042 & $-0,021$ & $-0,052$ & Thalassoma bifasciatum & $-0,077$ & 0,197 & $-0,138$ \\
\hline Odontoscion dentex & $-0,018$ & $-0,014$ & $-0,066$ & & & & \\
\hline Scarus iseri & $-0,026$ & $-0,08$ & $-0,317$ & & & & \\
\hline Scarus taeniopterus & $-0,054$ & $-0,001$ & $-0,314$ & & & & \\
\hline Scarus vetula & $-0,006$ & $-0,049$ & $-0,012$ & & & & \\
\hline Serranus tigrimus & 0,009 & 0,018 & $-0,02$ & & & & \\
\hline Sparisoma aurofrenatum & 0,021 & 0,111 & $-0,009$ & & & & \\
\hline Sparisoma viride & $-0,037$ & 0,087 & $-0,008$ & & & & \\
\hline Stegastes partitus & $-0,017$ & 0,062 & $-0,127$ & & & & \\
\hline Thalassoma bifasciatum & $-0,053$ & 0,942 & 0,175 & & & & \\
\hline
\end{tabular}

\section{Tabla 3}

Resultados de la prueba de Kruskal-Wallis para evaluar las diferencias entre los dos arrecifes estudiados respecto a componentes comunitarios

Results of Kruskal-Wallis test to evaluate the differences between the studied reefs with regard to community components

\begin{tabular}{lcccr}
\hline \multicolumn{1}{c}{ Variable } & Arrecife pétreo & Arrecife de octocorales & Estadigrafo H & Valor- $P$ \\
\hline Abundancia & $322,75 \pm 69,86$ & $38,42 \pm 11,49$ & 17,30 & $<0,0001$ \\
Diversidad & $4,11 \pm 0,22$ & $3,16 \pm 0,40$ & 15,88 & $<0,0001$ \\
Equitatividad & $0,80 \pm 0,05$ & $0,87 \pm 0,05$ & 7,05 & 0,0079 \\
Riqueza & $34,92 \pm 3,42$ & $12,92 \pm 3,12$ & 17,38 & $<0,0001$ \\
\hline
\end{tabular}


En el arrecife de corales blandos la mayoría de las especies son accidentales (53,7\%) o accesorias (21,9\%); mientras que sólo una cuarta parte de ellas se observó de manera constante $(24,4 \%)$. Las especies que estuvieron presentes en todos los meses del año fueron Aulostomus maculatus, Sparisoma aurofrenatum y Sparisoma viride.

El análisis de Kruskal-Wallis arrojó diferencias significativas respecto a la abundancia, la diversidad, la equitatividad y la riqueza de especies entre los dos arrecifes (Tabla 3).

\section{Discusión}

El año estudiado correspondió a un período particularmente distinto a lo que comúnmente sucede en la zona (Torres 1997, Cervigón 2005), debido a la poca influencia de los vientos alisios del noreste. Dichos vientos son, entre otros factores, el principal desencadenante de la surgencia estacional que típicamente afecta la región nororiental venezolana, con el consecuente incremento del plancton al igual que una disminución de la temperatura y de la transparencia del agua (Okuda 1978, Ferraz-Reyes 1989). Los valores altos de temperatura para marzo y abril $\left(28^{\circ} \mathrm{C}\right)$ registrados en el Bajo, destacan la naturaleza excepcional del período en el que se efectuó el estudio. Medina (2008), en cuatro sectores del archipiélago Las Caracas, cercanos al Bajo, y Silva (2009) en el Golfo de Cariaco, obtuvieron valores similares en estos meses para el mismo año. El estado de calma atípica observado pudiera haber sido responsable de la disminución en las capturas de sardina reportada por Lucentini (2006), debido a la escasez de su alimento.

No obstante, a pesar de la escasa influencia de los vientos del noreste, los resultados indican patrones de correlación que asocian la variación anual de la comunidad de peces con cambios estacionales de los parámetros ambientales. Estas asociaciones son más marcadas en el arrecife de octocorales que en el pétreo. El análisis de componentes principales, que evaluó la asociación de los parámetros ambientales y comunitarios a través de los meses, para el arrecife de octocorales, demostró una relación entre los meses de surgencia (diciembre y de enero a marzo), incluyendo el mes de julio, en el cual ha sido registrado un segundo pico de surgencia para la zona (Febres 1974). Al incrementarse los vientos disminuye la visibilidad y en este arrecife se incrementa la abundancia y la riqueza de especies, componentes comunitarios que igualmente se correlacionan entre sí. En este período, como ya ha sido ampliamente discutido por Cervigón (2005) aumenta el alimento disponible para los pequeños pelágicos migratorios, lo cual se evidencia en las agregaciones de Sardinella aurita y de Decapterus punctatus observadas en la zona estudiada. La ocurrencia de estos cardúmenes con fines alimenticios, es responsable de la disminución en la diversidad y en la equitatividad para los meses de surgencia. Las gráficas de la variación temporal de la abundancia y del número de especies refuerzan la relación positiva de la surgencia con la abundancia y la riqueza de especies de peces en el arrecife de octocorales. Este patrón es completamente opuesto a lo observado por Méndez et al. (2006) en un arrecife pétreo de Cautaro, localidad cercana en el Parque Nacional Mochima. Para dicho arrecife, los máximos de riqueza y abundancia suceden en los meses de calma, cuando no hay surgencia.

En el arrecife pétreo, a pesar de estar adyacente al arrecife de octocorales, las relaciones entre los parámetros ambientales y los componentes comunitarios son diferentes. En general, los meses del año no se asocian con una estacionalidad definida que pudiera marcar contrastes claros entre surgencia y calma. La abundancia relativa se relaciona con los meses de marzo y mayo. En marzo la ocurrencia de los cardúmenes ya señalados para el arrecife de octocorales de S. aurita y D. punctatus determina el mayor valor de abundancia; pero en mayo, la alta abundancia está determinada además por un elevado registro de individuos del pargo dientón Lutjanus griseus. La agregación para ese período en el Bajo Las Caracas se acerca a la temporada de desove que ha sido reportada para esta especie en Florida (Starck 1971) y en otras áreas de Venezuela (Cabrera et al. 1977), por lo que los autores realizaron capturas de algunos ejemplares, certificando el estado de avanzado desarrollo gonadal. En este sentido, la variación de la abundancia en el arrecife pétreo pareciera estar influenciada también por elementos reproductivos, además de la disponibilidad de alimento.

En los últimos años se han venido haciendo esfuerzos por certificar las agregaciones reproductivas de peces como una herramienta para delimitar áreas de protección especial. En el país, las agregaciones de peces con fines reproductivos no se encuentran debidamente documentadas. El Bajo Las Caracas se encuentra adyacente al límite norte del Parque Nacional Mochima pero fuera del área de protección y actualmente existe una propuesta para incluirlo dentro del Parque. La evidencia de la utilización de sus espacios como sitio de reproducción y desove para especies como el pargo dientón, con importancia económica y presión pesquera (Cabrera et al. 1977), sugieren la necesidad de su inclusión como zona protegida.

La riqueza en el arrecife pétreo se correlaciona positivamente con la visibilidad a diferencia de lo que sucede en el arrecife de octocorales. Tales resultados 
coinciden con lo planteado por Méndez et al. (2006) en Cautaro, quienes señalan que en la época en la cual baja la intensidad de los vientos y del oleaje y se incrementa la visibilidad, se crean condiciones favorables para la coexistencia de distintas especies en arrecifes someros. En estos meses, al disminuir los vientos, también se incrementa la temperatura (Ferraz-Reyes 1989). En el arrecife rocoso coralino del Bajo Las Caracas la temperatura tiende a asociarse de manera positiva con la riqueza, a pesar de que los valores de la prueba de Spearman no fueron significativos. Incremento de la riqueza cuando aumenta la temperatura ha sido registrado en arrecifes rocosos del pacífico mexicano (Pérez-España et al. 1996) y en sustratos coralinos de La Réunion (Letourneur \& Chabanet 1994), principalmente atribuido al reclutamiento en la temporada cálida y a una mayor disponibilidad de alimento en estas zonas.

Al comparar la variación mensual de las abundancias relativas de las especies no pelágicas entre los dos arrecifes, se observa que la abundancia relativa de cada especie permanece con menor variación a través de los meses del año en el arrecife pétreo; mientras que en el de octocorales la fluctuación mensual es mayor (Fig. 5). Esto se corrobora con el análisis de componentes principales, en el cual se relacionan los meses del año respecto a la abundancia de las especies no pelágicas, donde se denota una mayor asociación de los meses en el arrecife pétreo. En tal sentido, los peces del arrecife pétreo hacen un uso más frecuente del espacio arrecifal que los de los corales blandos. La presencia de determinados taxa durante los meses de muestreo apoya esta hipótesis, ya que la mayoría de las especies en los gorgonios hacen un uso accidental del espacio (53,66\%) o son accesorias (21,95\%); mientras que sólo una cuarta parte se observaron de manera constante (24,39\%).

La frecuencia de aparición encontrada en el arrecife pétreo del Bajo Las Caracas, que implica a 18 especies registradas en todos los muestreos, no coincide con lo reportado por Méndez et al. (2006) en Cautaro, donde sólo Thalassoma bifasciatum estuvo presente con alta frecuencia y dos especies más por sobre el 50\% de presencia en los meses evaluados: Microspathodon chrysurus y Diplodus argenteus. En cambio, en TurpialitoQuetepe, a pesar del bajo número de especies registrado (26), el 73,08\% de éstas fueron constantes, incluyendo a Scarus iseri, Halichoeres bivittatus, Elacatinus randalli y Haemulon aurolineatum (Núñez 2006).

Especies como Acanthurus coeruleus, Haemulon flavolineatum, Microspathodon chrysurus, Odontoscion dentex, Scarus vetula, Stegastes partitus, Mulloidichthys martinicus, Haemulon carbonarium y Cephalopholis cruentata, que estuvieron de manera constante sobre el arrecife pétreo a lo largo de todo el año de muestreo, no fueron observadas sobre los octocorales. Tal característica sugiere una alta especialización por estas especies que pareciera coincidir con las hipótesis determinísticas sobre la ocupación del espacio y la biodiversidad en los arrecifes pétreos (Ault \& Johnson 1998).

Los ecosistemas coralinos evaluados en este trabajo se encuentran a escasos metros de distancia, compartiendo las mismas fluctuaciones anuales de los parámetros ambientales. A pesar de ello, las variaciones estacionales de la abundancia, la frecuencia de aparición de las especies y las relaciones entre los componentes comunitarios y las variables fisicoquímicas medidas, fueron distintas entre ellos. Además, las diferencias significativas en todos los descriptores comunitarios (abundancia, diversidad, equitatividad y riqueza de especies) corrobora las diferencias ya registradas por Fariña et al. (2008) entre la composición de la ictiofauna del arrecife pétreo y la de los corales blandos y apoya la propuesta de que los arrecifes de octocorales sean considerados como un hábitat particularmente distinto dentro de la diversidad de ecosistemas del ambiente submarino venezolano.

\section{Agradecimientos}

A los copropietarios de la embarcación ‘Don Fernando’ y a los pescadores de 'La Anegada', por su apoyo en el trabajo de campo. Al profesor Alexander Barrios por su asesoría en los análisis estadísticos. A los evaluadores, por su aporte en el enriquecimiento del manuscrito.

\section{Literatura citada}

Ault TR \& CR Johnson. 1998. Spatially and temporally predictable fish communities on coral reefs. Ecological Monographs 68(1): 25-50.

Beukers IS \& GP Jones. 1998. Habitat complexity modifies the impact of piscivores on a coral reef fish population. Oecologia 114: 50-59.

Bouchon-Navarro Y, C Bouchon, M Louis \& P Legendre. 2005. Biogeographic patterns of coastal fish assemblages in the West Indies. Journal of Experimental Marine Biology and Ecology 315: 31-47.

Cabrera T, J Rosas \& J Millán. 1997. Reproducción y desarrollo larvario del pargo dientón (Lutjanus griseus L. 1758) (Pisces: Lutjanidae) cultivado en cautiverio. Caribbean Journal of Science 33(3-4): 239-245.

Caley MJ \& J St John. 1996. Refuge availability structures assemblages of tropical reef fishes. Journal of Animal Ecology 65: 414-428. 
CENAHP. 2007. Escala Beaufort-Intensidad del Viento. Centro Nacional de Alertas y Pronósticos Hidrometereológicos [online]<http://cenaph.gob.ve/leyendas_simbolos/ beaufort.php>.

Cervigón F. 1991. Los peces marinos de Venezuela. Vol I, 425 pp. Fundación Científica Los Roques, Caracas.

Cervigón F. 1993. Los peces marinos de Venezuela. Vol. II, 497 pp. Fundación Científica Los Roques, Caracas.

Cervigón F. 1994. Los peces marinos de Venezuela. Vol. III, 295 pp. Fundación Científica Los Roques, Caracas.

Cervigón F. 1996. Los peces marinos de Venezuela. Vol. IV, 254 pp. Fundación Científica Los Roques, Caracas.

Cervigón F. 2005. La ictiofauna marina de Venezuela: una aproximación ecológica. Boletín del Instituto Oceanográfico de Venezuela 44(1): 3-28.

Fariña A, E Méndez, S Sant \& E Zapata-Vívenes. 2008. Diferencias en la composición de especies de peces entre un arrecife rocoso-coralino y uno de octocorales en el Bajo Las Caracas, estado Sucre, Venezuela. Boletín del Centro de Investigaciones Biológicas 42(2): 165-185.

Febres GA. 1974. Circulación de las aguas superiores de la fosa de Cariaco en abril de 1979. Boletín del Instituto Oceanográfico de Venezuela 13(1-2): 79-86.

Ferraz-Reyes E. 1989. Influencia de los factores físicos en la distribución vertical de la biomasa fitoplanctónica en el Golfo de Cariaco (Venezuela). Boletín del Instituto Oceanográfico de Venezuela 28(1-2): 47-56.

Garpe KC \& MC Oehman. 2003. Coral and fish distribution patterns in Mafia Island Marine Park, Tanzania: fish-habitat interactions. Hydrobiologia 498(1-3): 191-211.

Ginsburg R. 2000. Protocolo AGRRA. Atlantic and Gulf Rapid Reef Assessment. Universidad de Miami, RSMAS/AGRR, Miami. [on-line] <http://coral.aoml.noaa.gov/agra> .

Grigg R. 1994. Effects of sewage discharge, fishing pressure and habitat complexity on coral ecosystems and reef fishes in Hawaii. Marine Ecology Progress Series 103: 25-34.

Humann P \& N Deloach. 2002. Reef Fish Identification, 512 pp. New World Publications, Jacksonville.

Krebs GJ. 1972. Ecology, 695 pp. Harper \& Row, New York.

Letorneur Y \& P Chabanet. 1994. Variations spatiotemporelles de l'ichtyofaune de platiers récifaux a La Réunion. Cybium 18(1): 25-38.
Lucentini O. 2006. Este mes se normaliza abastecimiento de sardina aseguró INAPESCA. Radio Nacional de VenezuelaNoticias.[on-line] <http://www.rnv.gov.ve/noticias/index> .

Medina M. 2008. Composición y estructura comunitaria de la ictiofauna en cuatro sectores de la isla Caracas Oeste, Parque Nacional Mochima, estado Sucre, Venezuela. Trabajo de Grado, Departamento de Biología, Universidad de Oriente, Cumaná, Venezuela, 87 pp.

Méndez E, LJ Ruiz, A Prieto, A Torres, A Fariña, S Sant, J Barrios \& B Marín. 2006. Fish community of a fringing reef at Mochima National Park, Venezuela. Ciencias Marinas 32(4): 683-693.

Núñez JG. 2006. Estructura de la comunidad íctica en un arrecife de coral franjeante de Turpialito-Quetepe, Golfo de Cariaco, estado Sucre, Venezuela. Trabajo de Grado, Departamento de Biología, Universidad de Oriente, Cumaná, 54 pp.

Okuda T. 1978. Condiciones hidrográficas de las aguas superficiales de la Fosa de Cariaco y áreas adyacentes. Boletín del Instituto Oceanográfico de Venezuela 21(1-2): 3-12.

Pérez-España H, F Galván-Magaña \& LAAbitia-Cárdenas. 1996. Variaciones temporales y espaciales en la estructura de la comunidad de peces de arrecifes rocosos del suroeste del Golfo de California, México. Ciencias Marinas 22(3): 273-294.

Ramírez-Gutiérrez M, M Tapia-García, E Ramos-Santiago \& R Ullo. 2007. Estructura comunitaria de peces en bahía San Agustín, Huatulco, México. Revista Chilena de Historia Natural 80: 419-430.

Risk MJ, DE McAllister \& L Behnken. 1998. Conservation of cold-and warm-water seafans: Threatened ancient gorgonian groves. Sea Wind 10(4): 20-22.

Silva F. 2009. Estructura de la comunidad íctica de una pradera de Thalassia en el saco del Golfo de Cariaco, estado Sucre, Venezuela. Trabajo de Grado, Departamento de Biología, Universidad de Oriente, Cumaná, Venezuela, 63 pp.

Starck WA II. 1971. Biology of the gray snapper Lutjanus griseus (Linnaeus) in the Florida Keys. En: Starck WA II \& $\mathrm{RE}$ Schroeder (eds). Investigations on the gray snapper, Lutjanus griseus. Studies in Tropical Oceanography 10: 11-150. University of Miami Press, Florida. 